\title{
Modern-day environmental factors in the pathogenesis of osteoarthritis
}

\section{Francis Berenbaum (D), Ian J. Wallace, Daniel E. Lieberman and David T. Felson}

\begin{abstract}
The prevalence of osteoarthritis $(\mathrm{OA})$ is rising for reasons that are not fully understood. In this Opinion article, we review the possibility that $O A$ is an evolutionary mismatch disease, which is a disease more common today than in the past because genes inherited from previous generations are inadequately or imperfectly adapted to modern environmental conditions. We focus on four major environmental factors in OA pathogenesis that have become ubiquitous within the past half-century: obesity, metabolic syndrome, dietary changes and physical inactivity. Because a cure for OA does not yet exist, prevention strategies that target these modifiable environmental factors are needed to curb further increases in OA prevalence.
\end{abstract}

To a large extent, osteoarthritis (OA) is a disease of old age, so its prevalence might be expected to be higher today than in the past simply because more people are living longer, especially in Europe, the United States and other developed nations ${ }^{1,2}$. However, evidence exists that increased longevity is probably not the sole reason for the high prevalence of OA. Wallace et al. ${ }^{3}$ traced long-term trends in the prevalence of knee OA in the United States using skeletal remains from 2,576 adults over the age of 50 , spanning from prehistoric hunter-gatherers to 21 st century city-dwellers. The results show that people who died since the mid-20th century were approximately twice as likely to have $\mathrm{OA}$ as those who died during earlier times, confirming expectations that the disease has become more common ${ }^{4-6}$. However, this spike in prevalence is apparent even after controlling for age in a generalized linear model, indicating the presence of additional major risk factors that have become ubiquitous only within the past half-century.

OA pathogenesis, like all disease aetiologies, involves interactions between genes and the environment ${ }^{7}$, but the increase in OA prevalence in just the past few generations indicates that environmental changes are a major contributor to the current high prevalence of OA. On this basis, OA seems to fit the definition of what evolutionary biologists refer to as a 'mismatch disease', that is, a condition that is more common today than in the past because the human body is not well adapted to certain features common to modern environments $s^{8,9}$. As there is no cure for OA, classification of it (at least partly) as a mismatch disease is of clinical consequence, as environmental factors are potentially modifiable targets for prevention.

Here, we discuss the effect of the environment on OA pathogenesis, focusing on factors that have become ubiquitous since the mid-20th century. To contextualize this evidence, we begin with an overview of the concept of mismatch diseases and conclude with a discussion of how understanding modern-day environmental factors is relevant to disease prevention.

\section{Mismatch diseases}

The concept of mismatch diseases derives from two basic principles of evolutionary biology: the theory of adaptation and the fact that interactions between genes and the environment are in constant flux ${ }^{8,9}$. Each individual inherits genes that interact with the environment, and most of these genes were favoured by natural selection because they improved the ability to survive and reproduce under particular environmental conditions. As a result, all organisms are adapted in varying degrees to aspects of the environment in which their ancestors existed, including associated diets and patterns of physical activity. When environments change, as they inevitably do, ancestral alleles once favoured by natural selection can become mismatched to features of the new environment. Ultimately, as a result of such mismatches, individuals have an increased susceptibility to illnesses that were once rare or nonexistent among earlier generations.

Mismatches between inherited genetic variants and changing environments are a fundamental engine of evolution ${ }^{10}$, but an abundance of evidence indicates that such mismatches are becoming more common and severe in humans owing to rapid environmental changes related to the cultural evolution of our species (reviewed elsewhere ${ }^{9}$ ).

Although humans have been huntergatherers for almost all of our $>200,000$-year evolutionary history, in just the past $\sim 12,000$ years, a large proportion of the global population has transitioned from being physically active hunter-gatherers, mainly consuming wild plants and animals, to being farmers settled in agricultural communities reliant on cereals and other domesticated foods to being post-industrial workers engaged in low levels of physical activity and eating highly processed foods. Although these changes in the environment, which have occurred in a blink of the eye in evolutionary time, have brought many benefits and comforts, they are also thought to be responsible for the emergence of a variety of mismatch diseases. For example, owing to the long evolutionary history of humans as physically active huntergatherers and consuming a diet rich in fibre but low in sugar ${ }^{9}$, the rising prevalence of type 2 diabetes is widely considered to be related to recent shifts towards physical inactivity and overconsumption of foods high in sugar but low in fibre, resulting in persistent positive energy balance, increased adiposity and chronic low-grade inflammation, which can lead to insulin insensitivity ${ }^{11}$.

When considering whether conditions such as $\mathrm{OA}$ are examples of mismatch 


\section{Box 1 | Effect of the obesity epidemic on osteoarthritis prevalence}

Although it is difficult to quantify precisely how much of the current prevalence of osteoarthritis $(\mathrm{OA})$ is attributable to any given environmental change, data from Wallace et al. ${ }^{3}$ provide a rough indication of the influence of the obesity epidemic on knee OA levels in the United States. Among individuals in their skeletal sample for which BMI at the time of death was documented, $25 \%$ of people who died in the past few decades were obese, compared with only $1 \%$ from earlier times, and individuals with obesity had a 2.2 times higher ( $95 \% \mathrm{Cl}$ 1.6-3.0) prevalence of knee OA than non-obese individuals. These data suggest that today obesity doubles the risk of knee $\mathrm{OA}$ in roughly 1 in 4 people over the age of 50, whereas only 1 in 100 people were at a similarly heightened risk of knee OA roughly a half-century ago. Although Wallace et al. ${ }^{3}$ were limited in their ability to assess the full effect of obesity on knee OA prevalence because BMI is a fairly inaccurate measure of excess adiposity and BMI was known only from individuals' time of death and not at the time they developed OA, these data provide strong evidence that the recent steep rise in obesity levels has led to substantially more people being at greater risk of developing knee OA.

diseases, however, caution is required, as the mismatch concept is often applied to a wide range of health disorders, in both the scientific literature and popular press, as more a matter of assumption than a hypothesis to be carefully tested. As with the so-called 'Paleo diet', overly simplistic claims are sometimes made about the potential health benefits associated with living more like our ancient ancestors and are based on misleading caricatures of past environments ${ }^{12}$ and the false assumption that humans evolved to be healthy? Clearly, not all features specific to modern environments interact negatively with the genes we inherit, and many environmental alterations can be beneficial, such as antibiotics, refrigeration or the use of casts for bone fractures. With this caveat in mind, we suggest two principal criteria for rigorously testing the mismatch hypothesis for diseases such as OA: first, that the disease is more prevalent today than among past populations after accounting for variation in lifespan and, second, that preventable contributors to the disease are more common in modern environments. Although OA is not a new disease and has been documented among Palaeolithic hunter-gatherers ${ }^{13}$ and Neolithic farmers ${ }^{14}$, the study by Wallace et al. ${ }^{3}$ and prior studies of smaller archaeological samples ${ }^{15,16}$ provide compelling evidence that $\mathrm{OA}$ meets the first criterion of a mismatch disease as being more prevalent today than in the past. Such studies, however, are retrospective and cannot identify all the causes of recent increases in OA. Nevertheless, evidence that the prevalence of OA in developed nations has spiked in the past half-century provides important clues about which preventable contributors to OA might be responsible, the most conspicuous candidates being obesity, metabolic syndrome, dietary changes and physical inactivity (FIG. 1).

\section{Mismatch factors}

Obesity. Obesity is commonly attributed as a source of mismatch diseases, as until modern times, most human bodies were rarely, if ever, exposed to long-term high levels of positive energy balance and hence rarely evolved adaptations to cope with the consequences of excess adipose tissue, especially visceral stores ${ }^{17}$. Unsurprisingly, obesity is a strong and well-established risk factor for $\mathrm{OA}^{18}$, especially knee $\mathrm{OA}^{19}$. Incidence of knee $\mathrm{OA}$ among adults aged $\geq 40$ years is reported to be approximately three times as frequent among obese individuals $(\mathrm{BMI} \geq 30)$ and five times as frequent among morbidly obese individuals (BMI $\geq 35)$ compared with individuals of a healthy weight $(\mathrm{BMI}<25)^{18}$. Given such a strong association, the rising prevalence of OA in developed nations is in some measure clearly attributable to the recent burgeoning obesity epidemic ${ }^{20}$ (BOX 1).

The link between obesity and knee OA is especially pernicious because it creates a vicious cycle in which pain from OA can greatly limit a person's physical activity, thus promoting further weight gain and weakening of muscles that stabilize and protect joints, which in turn can exacerbate pain and OA progression ${ }^{21}$. A negative feedback loop of this kind could just as easily be triggered by joint pain as by obesity, but evidence indicates that in the majority of cases, obesity precedes the onset of $\mathrm{OA}^{22,23}$. The driving, causal role of obesity in OA pathogenesis is further highlighted by evidence that most individuals with OA who have undergone bariatric surgery to induce weight loss experience a substantial reduction in joint pain and other symptoms $\mathrm{s}^{24,25}$. Evidence suggests that cartilage loss can be slowed if an obese person loses $10 \%$ or more of their original weight ${ }^{26}$. Weight loss may also reduce pain sensitivity and thereby contribute to pain relief $^{27}$.

Although the precise mechanisms by which obesity affects OA incidence are not completely understood, the longest-standing and perhaps most intuitive explanation is that obesity creates an abnormal loading environment for weight-bearing joints ${ }^{28}$.

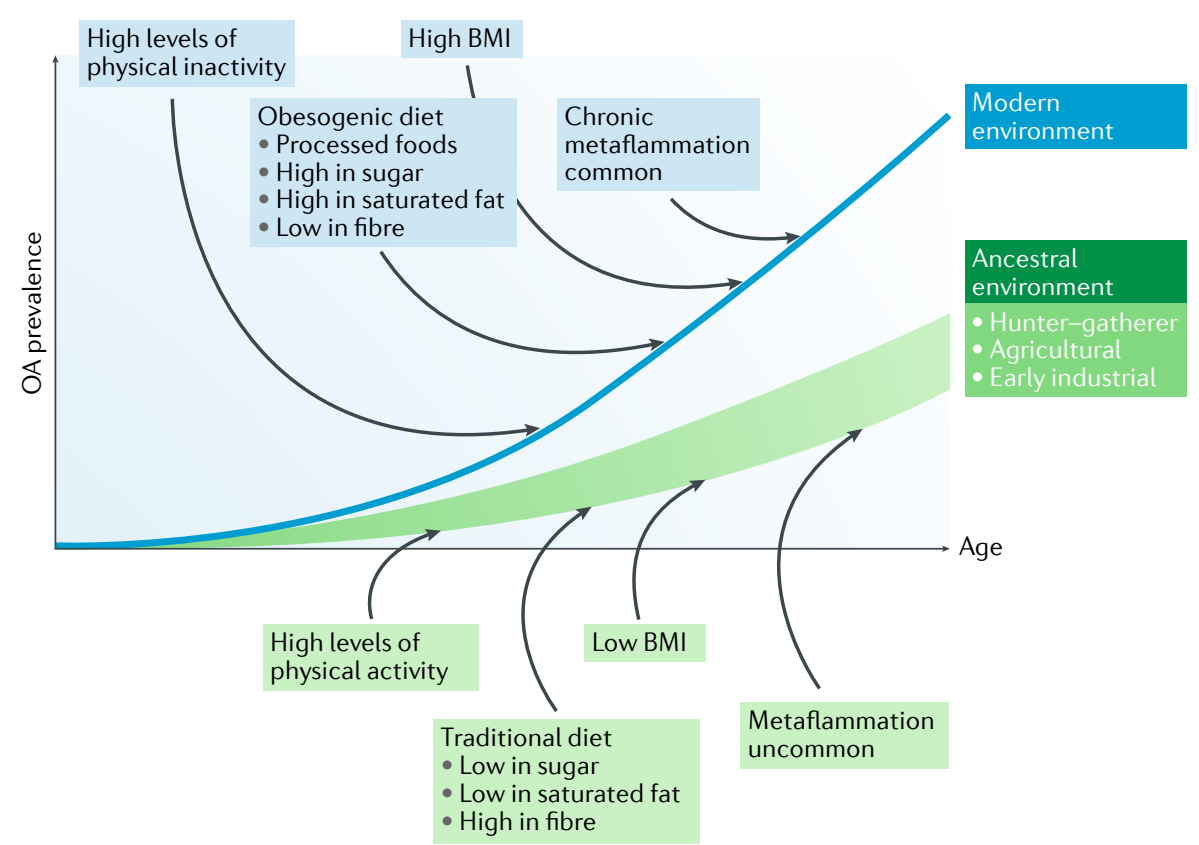

Fig. 1 | Model of osteoarthritis as a mismatch disease. In all populations, the prevalence of osteoarthritis $(\mathrm{OA})$ rises with age, but the hypothesis of mismatch predicts that prevalence at any given age is higher in modern environments because of high levels of obesity, chronic metaflammation and physical inactivity, and diets of processed foods that are rich in sugar and saturated fats and low in fibre. 


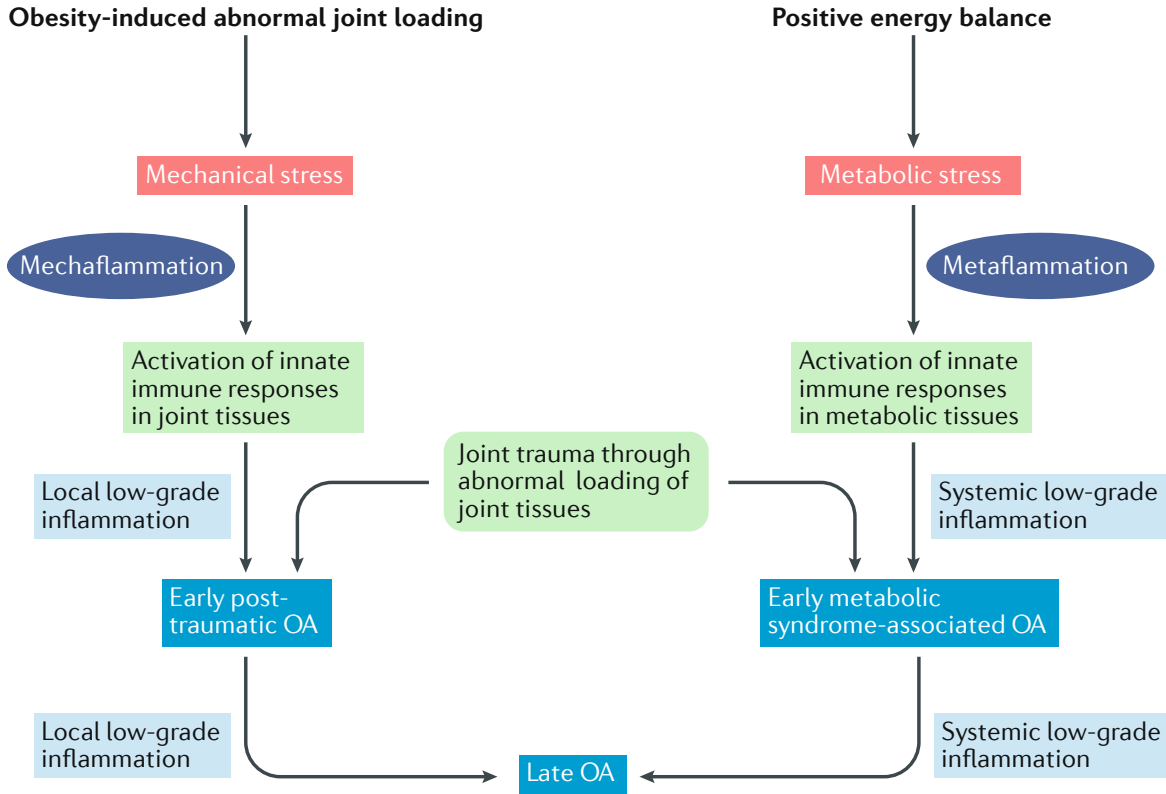

Fig. 2 | Mechaflammation versus metaflammation. Both osteoarthritis (OA) and obesity begin with activation of the innate immune system, which occurs by a local stimulus from joint tissues experiencing abnormal loading or a systemic stimulus from the adipose tissue. Triggering of innate immune responses can result in two types of low-grade inflammation, mechaflammation and metaflammation. Low-grade inflammation, in turn, weakens joint tissues, increasing their vulnerability to damage by subsequent loading and the initiation of $\mathrm{OA}$.

Loading per se is not bad for joints, as it is necessary for normal joint development and maintenance ${ }^{29,30}$, but some loads clearly have the potential to damage cartilage and other joint tissues and thus increase OA susceptibility, a fact highlighted by the strong link between traumatic injuries and $\mathrm{OA}^{31}$. The added body weight associated with obesity increases the magnitude of axial loads sustained by weight-bearing joints, which may impart some of the risk of OA caused by obesity. Among people with varus malalignment of the knee, such high-magnitude loads could be especially harmful, as they can magnify knee adduction moments ${ }^{32}$. Furthermore, low muscle strength relative to body weight may reduce the capacity of transarticular muscles to absorb shock and increase the rate and variability of joint loading ${ }^{33}$. A compromised ability to stabilize joints could cause forces to become concentrated in joint regions that are inadequately adapted for such loads and thus vulnerable to damage.

The primary result of aberrant loading of cartilage is damage to the structure of the cartilage matrix of collagen fibrils and proteoglycans $\mathrm{s}^{34,35}$. Cartilage degradation caused by abnormal loads may occur to some extent through wear and tear, but evidence suggests that the primary effect of such loads is to stimulate the production of metalloproteinases by chondrocytes and to activate these proteins in the matrix ${ }^{36}$. Abnormal loads trigger mechanoreceptors on the chondrocyte surface, which, in turn, trigger intracellular signalling pathways (for example, mitogen-activated protein kinase (MAPK) or nuclear factor- $\kappa \mathrm{B}$ $(\mathrm{NF}-\mathrm{kB})$ ) and the production of proinflammatory and catabolic mediators ${ }^{37,38}$. Matrix fragments released into the joint cavity can then provoke synoviocyte and macrophage responses and further release these pro-inflammatory and catabolic mediators, a process we refer to as mechaflammation ${ }^{39}$ (FIG. 2).

Mechanical factors are probably not the only contributors to obesity-induced OA, as obesity increases OA risk in not only weightbearing joints but also non-weight-bearing regions, such as hands ${ }^{40}$. The association between obesity and $\mathrm{OA}$ is generally stronger for weight-bearing than non-weight-bearing joints, but this difference in susceptibility across joints is evidence that the effect of obesity on OA involves complex interactions between mechanical and systemic factors ${ }^{41}$. Although much remains to be learned about these systemic factors, evidence indicates that a predominant source is adipose tissue, which produces and releases cytokines (including adipokines) into the bloodstream, many of which (such as IL-1, IL-6, IL-8, IFN $\gamma$, TNF, leptin and resistin) promote chronic low-grade inflammation, also termed metaflammation, for which the body is not well adapted ${ }^{42}$ (FIG. 2). Several of these cytokines have been shown experimentally to have an important function in initiating $\mathrm{OA}^{43}$. The adipokine leptin seems to be especially important in initiating $\mathrm{OA}$, as age-related knee OA does not occur in leptin-deficient obese mice ${ }^{44}$. The most direct pathway by which high levels of leptin and other cytokines in the bloodstream affect OA is by diffusing into the synovial fluid and locally activating proteolytic enzymes, such as matrix metalloproteinase 1 (MMP1), MMP3 and MMP13 (REF. ${ }^{45}$ ), which can trigger matrix degradation in cartilage and other joint tissues ${ }^{46}$. However, obesityinduced metaflammation may also affect OA more indirectly by modulating other critical metabolic factors, as discussed in the next section.

Metabolic syndrome. Another common source of mismatch diseases that also stems from excessive and long-term positive energy balance is metabolic syndrome, which is defined by a cluster of cardiometabolic factors that commonly accompany obesity, including central adiposity, dyslipidaemia, impaired fasting glucose levels and hypertension. Individuals with metabolic syndrome are at increased risk of a variety of health disorders, especially cardiovascular disease, type 2 diabetes and some cancers ${ }^{47}$. An abundance of evidence indicates that metabolic syndrome was once a rare (almost nonexistent) disease in nonindustrial populations ${ }^{48-50}$. Given the increase in prevalence of metabolic syndrome in developed nations, and an association with obesity, it is unsurprising that metabolic syndrome has been hypothesized to be a major risk factor for $\mathrm{OA}^{40,51}$.

Adipose-induced metaflammation is almost always associated with metabolic syndrome ${ }^{42}$ and strongly affects the metabolic dysregulation underlying multiple metabolic components ${ }^{52}$. In turn, these individual components of metabolic syndrome might affect the initiation or progression of $\mathrm{OA}^{53,54}$. For example, experimental evidence suggests that hyperglycaemia can have adverse effects on chondrocyte metabolism ${ }^{55-58}$, and type 2 diabetes can alter the structure of extracellular matrices, causing enrichment of advanced glycation endproducts (AGEs). In cartilage, AGEs stiffen the matrix, preventing optimal cushioning of the joints under a mechanical load ${ }^{59}$. Moreover, AGEs can signal chondrocytes through specific AGE receptors to increase 


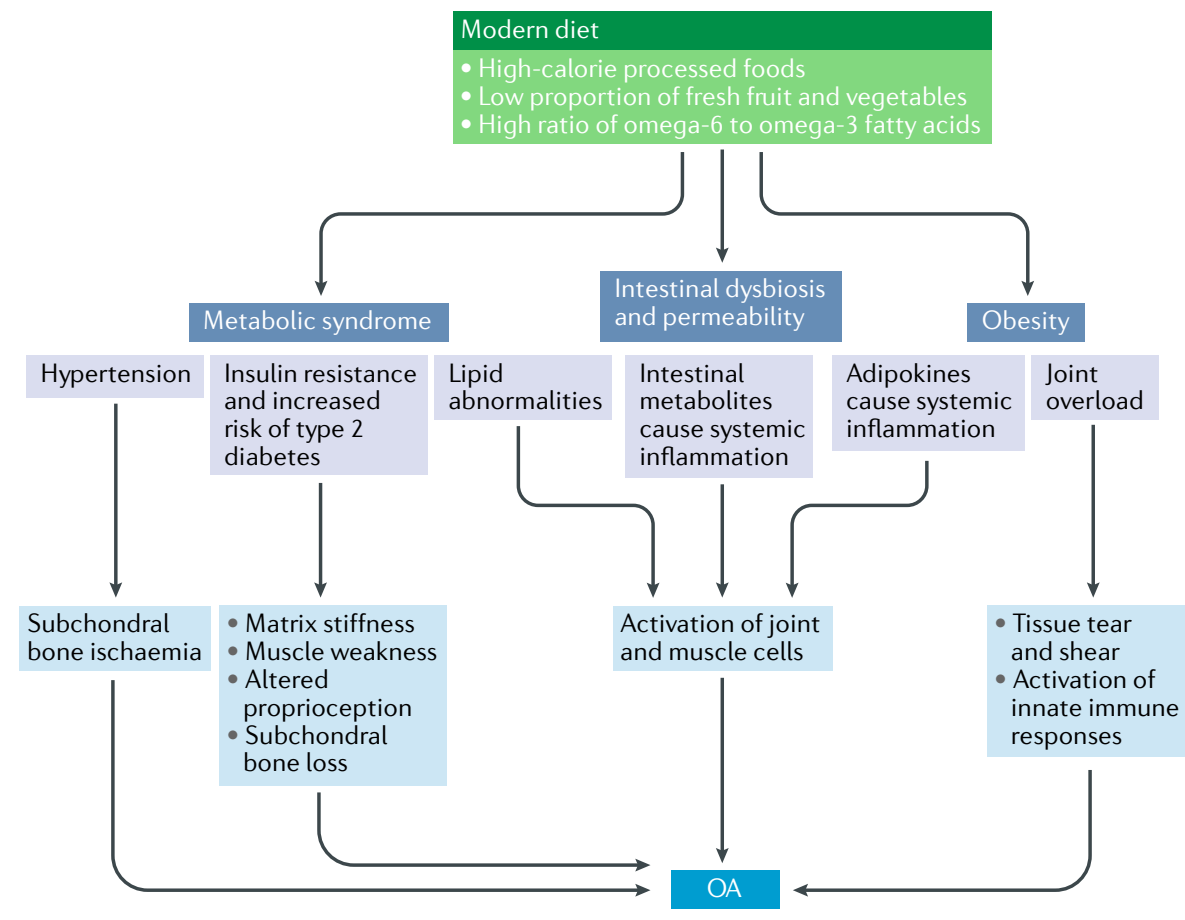

Fig. 3 | Diet as a mismatch factor. The deleterious effects of modern diets on osteoarthritis (OA) arise from increased adiposity and body mass, which leads to joint overload, a well-established risk factor for OA. Moreover, high caloric intake, a low proportion of fresh fruit and vegetables and a high ratio of omega- 6 to omega- 3 fatty acids, which are all hallmarks of a modern diet, participate in an increase in intestinal dysbiosis and permeability. These intestinal alterations increase systemic low-grade inflammation, a biological response suggested to trigger joint cell activation in OA. Modern diets are also considered the main cause of metabolic syndrome, which includes hypertension, insulin resistance and lipid abnormalities. Each of these pathologies could indirectly have an important function in OA pathogenesis through deleterious effects on joint tissues.

synthesis of metalloproteinases ${ }^{60}$ and thus should eventually lead to increased cartilage matrix degradation. Oxidized LDL, a pro-inflammatory peroxidized lipid detected at high concentration in plasma from patients with metabolic syndrome, can stimulate the production of reactive oxygen species by chondrocytes, propelling matrix degradation ${ }^{61}$. Hypertension might also be implicated in OA pathogenesis owing to induction of downstream tissue ischaemia. If ischaemia affects the blood vessels of the subchondral bone, the nutritional exchange between the subchondral bone and the cartilage might be compromised, resulting in altered metabolism of cells in the joints ${ }^{62}$.

Nevertheless, despite experimental evidence of multiple potential pathways linking metabolic syndrome and $\mathrm{OA}$, data from human studies are conflicting, with most studies showing no association of metabolic syndrome with knee OA after taking into account BMI. For example, in a study of 991 individuals, metabolic syndrome was strongly associated with incident knee OA, but after controlling for body weight, the associations disappeared ${ }^{63}$. Other studies, however, have found that hand OA (but not knee $\mathrm{OA}$ ) is strongly associated with metabolic syndrome even after adjusting for body weight ${ }^{41}$. Interestingly, people with hypertension have been shown to have an elevated risk of knee OA independent of obesity ${ }^{63}$, and OA prevalence was higher among people with type 2 diabetes than among people without diabetes, independent of weight differences ${ }^{64}$. Moreover, an MRI study indicates that patients with type 2 diabetes have accelerated knee cartilage matrix degeneration compared with individuals without diabetes, even after correcting for ethnicity, age, sex, baseline BMI and severity of $\mathrm{OA}$ as measured by baseline Kellgren - Lawrence score ${ }^{65}$. Although experimental research and some human studies provide evidence that individual components of metabolic syndrome (aside from adiposity) contribute to $\mathrm{OA}$ pathogenesis, more data are necessary to resolve the degree to which the current prevalence of $\mathrm{OA}$ is attributable to modern increases in metabolic syndrome prevalence.
Dietary changes. The increase in OA prevalence in developed nations raises the question of whether changes in diet cause mismatches that contribute to OA. Modern diets in many developed countries differ from those of earlier generations in being substantially more energy dense and processed, with added sugar, salt and saturated fats but less fibre, fresh fruits and vegetables. ${ }^{9}$ These dietary shifts almost certainly affect $\mathrm{OA}$ risk by promoting prolonged positive energy balance and excess adiposity but also perhaps by increasing the probability of hyperglycaemia, dyslipidaemia and hypertension.

Aside from promoting metabolic dysregulation, however, modern dietary changes potentially affect $\mathrm{OA}$ risk in other ways. An additional dietary factor of particular relevance is a reduced intake of antioxidants ${ }^{6}{ }^{6}$. Reactive oxygen species are involved in chondrocyte senescence, extracellular matrix degradation, synovial inflammation and subchondral bone alteration ${ }^{67}$. Diets in many developed nations are characterized by an increase in the ratio of pro-inflammatory omega- 6 fatty acids to anti-inflammatory omega- 3 polyunsaturated fatty acids ${ }^{68}$. However, evidence that this imbalance contributes to disease remains a contested point of debate. In one study, supplementing the diet with omega-3 fatty acids reduced the severity of posttraumatic $\mathrm{OA}$ in mice and limited attendant synovitis ${ }^{69}$, whereas in another study, dietary enrichment of omega- 3 fatty acids did not reduce the onset of knee $\mathrm{OA}$ in mice ${ }^{70}$. In humans, the effect of omega- 3 fatty acid supplements in OA trials has not been reported to affect joint pain ${ }^{71,72}$. Moreover, sulforaphane, an isothiocyanate abundant in broccoli, decreased the severity of OA in mice, possibly by protecting against damage from reactive oxygen species ${ }^{73,74}$; plans now exist to test the consumption of broccoli in an OA clinical trial ${ }^{75}$. Conflicting evidence exists regarding the effect of the antioxidant vitamin $\mathrm{C}$ on $\mathrm{OA}$ in humans ${ }^{76-79}$, with experiments in mice, rats and guinea pigs showing that vitamin $\mathrm{C}$ may increase $\mathrm{OA}$ risk $^{80}$. On the other hand, vitamin $\mathrm{K}$, present in green leafy vegetables like spinach, kale and broccoli, is a necessary cofactor for the $\gamma$-carboxylation of some calcium-binding proteins, including matrix gla protein, a vitamin $\mathrm{K}$-dependent mineralization inhibitor expressed in human articular cartilage. Many human observational studies have reported that vitamin $\mathrm{K}$ deficiency increases the risk of $\mathrm{OA}^{81-83}$, but clinical trials testing vitamin $\mathrm{K}$ treatment have not yet been performed. Experimental 


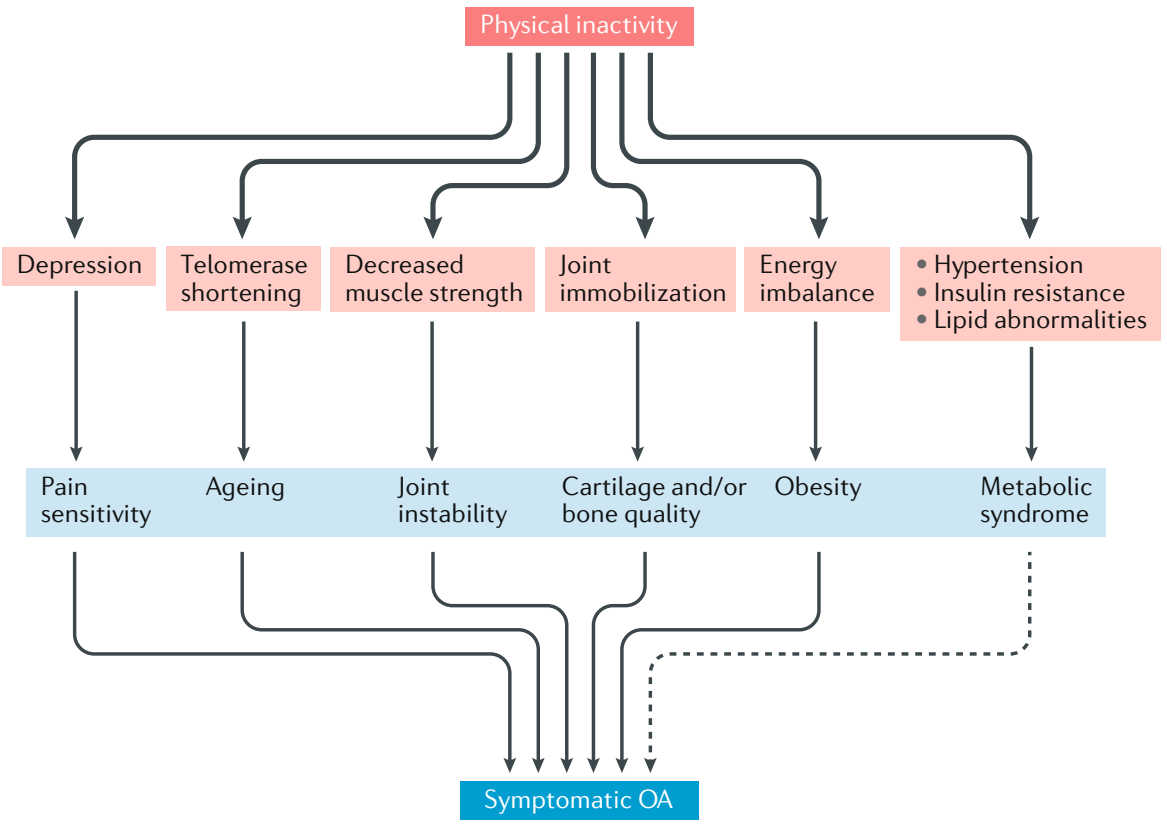

Fig. 4 | Physical inactivity as a mismatch factor. A sedentary lifestyle and physical inactivity might initiate and aggravate osteoarthritis (OA) and its symptoms via a variety of pathways.

studies point to other dietary factors that are potentially implicated in OA but have not yet been carefully examined in human studies (FIG. 3). Some groups have shown that dietary high-fat overload can increase the severity of post-traumatic OA in mice and rats $^{69,84,85}$. Interestingly, for the same quantity of calories, severity of OA was exacerbated by a diet rich in saturated as opposed to unsaturated fatty acids ${ }^{69}$.

Obesity and ageing are associated with intestinal dysbiosis that may cause metabolic age-related chronic diseases ${ }^{86,87}$. The function of the diet in modulating the composition and metabolic activity of the intestinal microbiome is now recognized ${ }^{88}$. Diffusion of biologically active metabolites (such as acetate, propionate and butyrate) and lipopolysaccharide, a microbial cell wall constituent, from the gut to bloodstream related to increased intestinal permeability and dysbiosis in patients with obesity is associated with low-grade systemic inflammation ${ }^{89-91}$. Although evidence that these dysbiosis-derived metabolites have a direct pathophysiological function in OA is lacking, the results of some experimental studies are consistent with this hypothesis ${ }^{92,93}$. One important dietary factor that modifies the gut microbiota is fibre; changes in the intestinal microbiome might be related to a paucity of fibre in the modern diet. In two cohorts, volunteers in the highest quartile of total fibre intake had lower rates of new-onset symptomatic OA than those in the lowest quartile of total fibre vigorous physical activity and yet had lower prevalence of $\mathrm{OA}^{98,99}$. Whether people today are, on average, more susceptible to injury and post-traumatic OA than in the past is highly speculative.

Although trauma unquestionably increases OA risk, a more likely contributor to the increased prevalence of OA is physical inactivity, which has become epidemic in the past few decades, especially in many developed nations ${ }^{100}$. Pathways by which physical inactivity can increase OA risk include indirect promotion of obesity and metaflammation, depression ${ }^{101}$ or telomere shortening ${ }^{102}$ (FIG. 4). However, physical inactivity might also contribute to OA pathogenesis directly. Because the musculoskeletal system, like many physiological systems, evolved to require biophysical stimuli from the environment to adjust capacity to demand ${ }^{103}$, mechanical loads engendered by activity are critical to the development and maintenance of optimal structure and strength of joint tissues and their surrounding muscles ${ }^{29,104}$. Moreover, a reduction in loading as a result of a physically inactive lifestyle might cause formation of weaker and less stable joints that are more susceptible to damage and deterioration ${ }^{105,106}$. In other words, physical inactivity leads to an absence of normal demand, whereby individuals are unlikely to attain or maintain normal joint capacity.

To illustrate this 'use it or lose it' principle in cartilage, patients with paralysed limbs exhibit marked knee cartilage thinning ${ }^{106,107}$, whereas MRI studies have shown that people who regularly engage in weight-bearing exercise maintain thicker cartilage, and in one study, these individuals were even noted to have fewer cartilage defects, than people who are physically inactive ${ }^{108-110}$. Animal experiments have yielded similar findings: disuse experiments (for example, rodent limb immobilization or unloading) consistently demonstrate multiple catabolic effects on joint tissues, including thinning of all cartilage layers, decreased cartilage proteoglycan content by increased expression of metalloproteinases and demineralization of subchondral bone by osteoclast activation ${ }^{111-114}$. By contrast, a meta-analysis of exercise in various animal species showed that, compared with animals on a moderate daily exercise regimen, nonexercising control animals had thinner knee cartilage with lower aggrecan content ${ }^{115}$. Thinner cartilage with lower aggrecan content is not necessarily osteoarthritic cartilage (for example, paralysed limbs 
rarely get $\mathrm{OA}$ ), but it is biomechanically vulnerable cartilage ${ }^{116}$.

Even if physical inactivity is detrimental to joint health, this does not mean that all forms of physical activity are beneficial for joints. As already discussed, some types of loading can threaten the integrity of joint tissues, and loads that are extreme or otherwise abnormal, either in terms of magnitude, frequency or some other parameter, which are produced by active lifestyles through occupation (for example, jobs requiring frequent knee bending) or recreation (for example, sports injuries) can culminate in damaged joints that are more prone to OA. Thus, risk of OA is probably increased by both extreme physical inactivity and activity ${ }^{35,117}$. However, although considerable research and clinical attention has been paid to the potential negative consequences of some types of physical activity for joint health, greater attention ought to be devoted to understanding the degree to which decreases in physical activity underlie high levels of OA today.

\section{Conclusions}

Although the causes of the high and rising prevalence of OA are still not entirely understood, one important conclusion of this article is that OA fits the criteria of a mismatch disease in that the current $\mathrm{OA}$ prevalence seems to be partly attributable to environmental risk factors that have

\footnotetext{
Glossary

Adaptation

A phenotypic trait favoured by natural selection

because it improves an organism's ability to survive and reproduce.

Developed nations

Wealthy countries with post-industrial economies and advanced technological infrastructure.

Hunter-gatherers

People who subsist on foraged wild plants and hunted wild animals, in contrast to agriculturalists who subsist mainly on domesticated plants and animals.

Knee adduction moments

Dynamic rotational forces (torques) that act on the knee in the coronal plane, applying a compressive force to the medial side of the knee.

Kellgren-Lawrence score

A common method of classifying the severity of knee osteoarthritis using radiography.

Mechaflammation

Focal inflammation owing to a local mechanical insult.

Metaflammation

Chronic, low-grade, metabolic and systemic

inflammation.

Varus malalignment

A deformity of the knee in which the distal leg is angled medially in relation to the axis of the thigh, resulting in a bowlegged appearance.
}

become amplified in the modern world. These factors probably include obesity, metabolic syndrome, dietary changes and physical inactivity. A second and even more important conclusion is that, although OA risk is influenced by intrinsic factors such as age and genetics, OA is partly a mismatch disease affected by modifiable factors, indicating substantial potential for prevention. This is a critical insight given that available nonsurgical treatments for OA provide relief from symptoms only, and no disease-modifying drugs exist. In short, although OA may seem to be mainly a disease of old age, from an evolutionary perspective, it is not age per se that causes the disease but an accumulation of joint tissue deterioration arising from interactions between the genes we inherited from our ancestors and the environments - many of them novel yet modifiable - that we encounter as we grow older.

Because of human evolutionary origins as physically active hunter-gatherers on the margin of energy balance, human joints probably evolved to require routine mechanical loading in the absence of adiposity-induced metaflammation to grow and function optimally with age. However, even if OA is partly a mismatch disease, the disease would not cease to occur even if everyone on the planet adjusted their lifestyles to more closely match the conditions for which the human musculoskeletal system is adapted. As the incidence of $\mathrm{OA}$ in prehistoric populations testifies, trauma and other risk factors have always predisposed some people to OA. Nevertheless, on the basis of the available evidence reviewed here, it seems unlikely that the OA epidemic will be curbed without at least beginning to reverse declines in physical activity levels and the quality of our diets, along with attendant effects on obesity and metabolic dysregulation. How to promote such lifestyle changes is a major challenge.

Francis Berenbaum iD ${ }^{1}$, , Ian J. Wallace ${ }^{2}$,

Daniel E. Lieberman ${ }^{2}$ and David T. Felson ${ }^{3,4}$

'Sorbonne Université, INSERM, DHU i2B, AP-HP, Hôpital Saint-Antoine, Paris, France.

${ }^{2}$ Department of Human Evolutionary Biology, Peabody Museum, Harvard University, Cambridge, MA, USA.

${ }^{3}$ Clinical Epidemiology Unit, Boston University School of Medicine, Boston, MA, USA.

${ }^{4}$ NIHR Manchester Musculoskeletal Biomedical Research Centre, University Hospitals NHS Foundation Trust, Manchester Academic Health Science Centre, Manchester, UK.

*e-mail: francis.berenbaum@aphp.fr

https://doi.org/10.1038/s41584-018-0073-x

Published online: 12 September 2018
1. Felson, D. T. et al. Osteoarthritis: new insights. Part 1: the disease and its risk factors. Ann. Intern. Med. 133 635-646 (2000).

2. Bijlsma, J. W., Berenbaum, F. \& Lafeber, F. P. Osteoarthritis: an update with relevance for clinical practice. Lancet 377, 2115-2126 (2011).

3. Wallace, I. J. et al. Knee osteoarthritis has doubled in prevalence since the mid-20th century. Proc. Natl Acad. Sci. USA 114, 9332-9336 (2017).

4. Nguyen, U. S. et al. Increasing prevalence of knee pain and symptomatic knee osteoarthritis: survey and cohort data. Ann. Intern. Med. 155, 725-732 (2011).

5. GBD 2013 DALYs and HALE Collaborators. Global, regional, and national disability-adjusted life years (DALYs) for 306 diseases and injuries and healthy life expectancy (HALE) for 188 countries, 1990-2013: quantifying the epidemiological transition. Lancet 386, 2145-2191 (2015)

6. Kiadaliri, A. A., Lohmander, L. S., Moradi-Lakeh, M., Petersson, I. F. \& Englund, M. High and rising burden of hip and knee osteoarthritis in the Nordic region, 1990-2015. Acta Orthop. 89, 177-183 (2017).

7. Sandell, L. J. Etiology of osteoarthritis: genetics and synovial joint development. Nat. Rev. Rheumatol. 8, 77-89 (2012)

8. Gluckman, P. D. \& Hanson, M. A. Mismatch: The Lifestyle Diseases Timebomb (Oxford Univ. Press, 2013).

9. Lieberman, D. E. The Story of the Human Body: Evolution, Health and Disease (Pantheon Books, 2013)

10. Rose, M. R. \& Lauder, G. V. Adaptation (Academic Press, 1996).

11. Menke, A., Casagrande, S., Geiss, L. \& Cowie, C. C. Prevalence of and trends in diabetes among adults in the United States, 1988-2012. JAMA 314, 1021-1029 (2015).

12. Zuk, M. Paleofantasy: What Evolution Really Tells Us About Sex, Diet, and How We Live (W. H. Norton, 2014).

13. Pontzer, $\mathrm{H}$. et al. Locomotor anatomy and biomechanics of the Dmanisi hominins. J. Hum. Evol. 58, 492-504 (2010).

14. Larsen, C. S. et al. Bioarchaeology of Neolithic Çatalhöyük: lives and lifestyles of an early farming society in transition. J. World Prehistory 28, 27-68 (2015).

15. Rogers, J. \& Dieppe, P. Is tibiofemoral osteoarthritis in the knee joint a new disease? Ann. Rheum. Dis. $\mathbf{5 3}$ 612-613 (1994).

16. Inoue, K. et al. Prevalence of large-joint osteoarthritis in Asian and Caucasian skeletal populations. Rheumatology 40, 70-73 (2001).

17. Lieberman, D. E. Is exercise really medicine? An evolutionary perspective. Curr. Sports Med. Rep. 14, 313-319 (2015)

18. Reyes, C. et al. Association between overweight and obesity and risk of clinically diagnosed knee, hip, and hand osteoarthritis: a population-based cohort study. Arthritis Rheum. 68, 1869-1875 (2016).

19. Felson, D. T. Epidemiology of hip and knee osteoarthritis. Epidemiol. Rev. 10, 1-28 (1988).

20. Ng, M. et al. Global, regional, and national prevalence of overweight and obesity in children and adults during 1980-2013: a systematic analysis for the Global Burden of Disease Study 2013. Lancet 384, 766-781 (2014).

21. Wluka, A. E., Lombard, C. B. \& Cicuttini, F. M. Tackling obesity in knee osteoarthritis. Nat. Rev. Rheumatol. 9 225-235 (2013)

22. Felson, D. T., Anderson, J. J., Naimark, A., Walker, A. M \& Meenan, R. F. Obesity and knee osteoarthritis. The Framingham Study. Ann. Intern. Med. 109, 18-24 (1988).

23. Gelber, A. C. et al. Body mass index in young men and the risk of subsequent knee and hip osteoarthritis. Am. J. Med. 107, 542-548 (1999).

24. Richette, P. et al. Benefits of massive weight loss on symptoms, systemic inflammation and cartilage turnover in obese patients with knee osteoarthritis. Ann. Rheum. Dis. 70, 139-144 (2011).

25. King, W. C. et al. Change in pain and physical function following bariatric surgery for severe obesity. JAMA 315, 1362-1371 (2016).

26. Gersing, A. S. et al. Is weight loss associated with less progression of changes in knee articular cartilage among obese and overweight patients as assessed with MR imaging over 48 months? Data from the Osteoarthritis Initiative. Radiology 284, 508-520 (2017).

27. Stefanik, J. J. et al. Changes in pain sensitization after bariatric surgery. Arthritis Care Res. https://doi. org/10.1002/acr.23513 (2018).

28. Wearing, S. C., Hennig, E. M., Byrne, N. M., Steele, J. R. $\&$ Hills, A. P. Musculoskeletal disorders associated with obesity: a biomechanical perspective. Obes. Rev. 7, 239-250 (2006). 
29. Griffin, T. M. \& Guilak, F. The role of mechanical loading in the onset and progression of osteoarthritis. Exerc. Sport Sci. Rev. 33, 195-200 (2005).

30. Giorgi, M., Carriero, A., Shefelbine, S. J. \& Nowlan, N. C. Effects of normal and abnormal loading conditions on morphogenesis of the prenatal hip joint: application to hip dysplasia. J. Biomechan. 48, 3390-3397 (2015).

31. Felson, D. T. Osteoarthritis as a disease of mechanics. Osteoarthritis Cartilage 21, 10-15 (2013).

32. Felson, D. T., Goggins, J., Niu, J., Zhang, Y. \& Hunter, D. J. The effect of body weight on progression of knee osteoarthritis is dependent on alignment. Arthritis Rheum. 50, 3904-3909 (2004).

33. Slemenda, C. et al. Reduced quadriceps strength relative to body weight: a risk factor for knee osteoarthritis in women? Arthritis Rheum 41 1951-1959 (1998)

34. Buckwalter, J. A. \& Mankin, H. J. Articular cartilage: tissue design and chondrocyte-matrix interactions. Instr. Course Lect. 47, 477-486 (1998).

35. Sanchez-Adams, J., Leddy, H. A., McNulty, A. L., O'Conor, C. J. \& Guilak, F. The mechanobiology of articular cartilage: bearing the burden of osteoarthritis. Curr. Rheumatol. Rep. 16, 451-451 (2014).

36. Pottie, P. et al. Obesity and osteoarthritis: more complex than predicted! Ann. Rheum. Dis. 65, 1403-1405 (2006)

37. Houard, X., Goldring, M. B. \& Berenbaum, F. Homeostatic mechanisms in articular cartilage and role of inflammation in osteoarthritis. Curr Rheumatol. Rep. 15, 375-375 (2013).

38. Millward-Sadler, S. J. \& Salter, D. M. Integrindependent signal cascades in chondrocyte mechanotransduction. Ann. Biomed. Engineer. 32, 435-446 (2004)

39. Loeser, R. F., Goldring, S. R., Scanzello, C. R. $\overline{1}$ Goldring, M. B. Osteoarthritis: a disease of the joint as an organ. Arthritis Rheum. 64, 1697-1707 (2012)

40. Yusuf, E. et al. Association between weight or body mass index and hand osteoarthritis: a systematic review. Ann. Rheum. Dis. 69, 761-765 (2010)

41. Visser, A. W. et al. The relative contribution of mechanical stress and systemic processes in different types of osteoarthritis: the NEO study. Ann. Rheum. Dis. 74, 1842-1847 (2015).

42. Hotamisligil, G. S. Inflammation, metaflammation and immunometabolic disorders. Nature 542, 177-185 (2017).

43. Berenbaum, F., Eymard, F. \& Houard, X Osteoarthritis, inflammation and obesity. Curr. Opin. Rheumatol. 25, 114-118 (2013).

44. Griffin, T. M., Huebner, J. L., Kraus, V. B. \& Guilak, F Extreme obesity due to impaired leptin signaling in mice does not cause knee osteoarthritis. Arthritis Rheum. 60, 2935-2944 (2009).

45. Berenbaum, F. Osteoarthritis as an inflammatory disease (osteoarthritis is not osteoarthrosis!). Osteoarthritis Cartilage 21, 16-21 (2013).

46. Francisco, V. et al. Biomechanics, obesity, and osteoarthritis. The role of adipokines: when the levee breaks. J. Orthop. Res. 36, 594-604 (2018).

47. Eckel, R. H., Grundy, S. M. \& Zimmet, P. Z. The metabolic syndrome. Lancet 365, 1415-1428 (2005)

48. Kaplan, H. et al. Coronary atherosclerosis in indigenous South American Tsimane: a cross-sectional cohort study, Lancet 389, 1730-1739 (2017).

49. Kaminer, B. \& Lutz, W. P. Blood pressure in Bushmen of the Kalahari Desert. Circulation 22, 289-295 (1960).

50. Raichlen, D. A et al. Physical activity patterns and biomarkers of cardiovascular disease risk in huntergatherers. Am. J. Hum. Biol. 29, e22919 (2017).

51. Moore, J. X., Chaudhary, N. \& Akinyemiju, T. Metabolic syndrome prevalence by race/ethnicity and sex in the United States, National Health and Nutrition Examination Survey, 1988-2012. Prev. Chron. Dis. 14, E24 (2017)

52. Lee, Y. S., Wollam, J. \& Olefsky, J. M. An integrated view of immunometabolism. Cell 172, 22-40 (2018)

53. Berenbaum, F., Griffin, T. M. \& Liu-Bryan, R. Metabolic regulation of inflammation in osteoarthritis. Arthritis Rheumatol. 69, 9-21 (2017).

54. Zhuo, Q., Yang, W., Chen, J. \& Wang, Y. Metabolic syndrome meets osteoarthritis. Nat. Rev. Rheumatol. 8, 729 (2012)

55. Rosa, S. C. et al. Impaired glucose transporter-1 degradation and increased glucose transport and oxidative stress in response to high glucose in chondrocytes from osteoarthritic versus norma human cartilage. Arthritis Res. Ther. 11, R80 (2009)
56. Rosa, S. C. et al. Role of glucose as a modulator of anabolic and catabolic gene expression in normal and osteoarthritic human chondrocytes. J. Cell. Biochem. 112, 2813-2824 (2011).

57. Vaamonde-Garcia, C. et al. The nuclear factorerythroid 2-related factor/heme oxygenase- 1 axis is critical for the inflammatory features of type 2 diabetes-associated osteoarthritis. J. Biol. Chem. 292 14505-14515 (2017).

58. Berenbaum, F. Diabetes-induced osteoarthritis: from a new paradigm to a new phenotype. Ann. Rheum. Dis. 70, 1354-1356 (2011)

59. Shane Anderson, A. \& Loeser, R. F. Why is osteoarthritis an age-related disease? Best practice and research. Clin. Rheumatol. 24, 15-26 (2010)

60. Steenvoorden, M. M. et al. Activation of receptor for advanced glycation end products in osteoarthritis leads to increased stimulation of chondrocytes and synoviocytes. Arthritis Rheum. 54, 253-263 (2006).

61. de Munter, W., van der Kraan, P. M., van den Berg, W. B. $\&$ van Lent, P. L. High systemic levels of low-density lipoprotein cholesterol: fuel to the flames in inflammatory osteoarthritis? Rheumatology 55 , 16-24 (2016)

62. Conaghan, P. G., Vanharanta, H. \& Dieppe, P. A. Is progressive osteoarthritis an atheromatous vascular disease? Ann. Rheum. Dis. 64, 1539-1541 (2005)

63. Niu, J., Clancy, M., Aliabadi, P., Vasan, R. \& Felson, D. T. Metabolic syndrome, its components, and knee osteoarthritis: The Framingham Osteoarthritis Study. Arthritis Rheumatol. 69, 1194-1203 (2017).

64. Louati, K., Vidal, C., Berenbaum, F. \& Sellam, J. Association between diabetes mellitus and osteoarthritis: systematic literature review and meta-analysis. RMD Open 1 , e000077 (2015).

65. Neumann, J. et al. Type 2 diabetes patients have accelerated cartilage matrix degeneration compared to diabetes free controls: data from the Osteoarthritis Initiative. Osteoarthritis Cartilage 26, 751-761 (2018)

66. Ruiz-Nunez, B., Pruimboom, L., Dijck-Brouwer, D. A. \& Muskiet, F. A. Lifestyle and nutritional imbalances associated with Western diseases: causes and consequences of chronic systemic low-grade inflammation in an evolutionary context. J. Nutr. Biochem. 24, 1183-1201 (2013).

67. Lepetsos, P \& Papavassiliou, A G ROS/oxidative stress signaling in osteoarthritis. Biochim. Biophys. Acta 1862, 576-591 (2016).

68. Simopoulos, A. P. An increase in the omega-6/omega-3 fatty acid ratio increases the risk for obesity. Nutrients 8, 128 (2016)

69. Wu, C. L. et al. Dietary fatty acid content regulates wound repair and the pathogenesis of osteoarthritis following joint injury. Ann. Rheum. Dis. 74 2076-2083 (2015).

70. Cai, A. et al. Metabolic enrichment of omega-3 polyunsaturated fatty acids does not reduce the onset of idiopathic knee osteoarthritis in mice. Osteoarthritis Cartilage 22, 1301-1309 (2014).

71. Senftleber, N. et al. Marine oil supplements for arthritis pain: a systematic review and meta-analysis of randomized trials. Nutrients 9, 42 (2017).

72. Hill, C. L. et al. Fish oil in knee osteoarthritis: a randomised clinical trial of low dose versus high dose. Ann. Rheum. Dis. 75, 23-29 (2016)

73. Davidson, R. K. et al. Sulforaphane represses matrixdegrading proteases and protects cartilage from destruction in vitro and in vivo. Arthritis Rheum. 65 3130-3140 (2013)

74. Berenbaum, F. Does broccoli protect from osteoarthritis? Joint Bone Spine 81, 284-286 (2014).

75. Davidson, R. et al. Isothiocyanates are detected in human synovial fluid following broccoli consumption and can affect the tissues of the knee joint. Sci. Rep. 7 3398 (2017)

76. McAlindon T E et al. Do antioxidant micronutrients protect against the development and progression of knee osteoarthritis? Arthritis Rheum. 39, 648-656 (1996).

77. Sanghi, D. et al. Elucidation of dietary risk factors in osteoarthritis knee - a case-control study. J. Am. College Nutr. 34, 15-20 (2015).

78. Peregoy, J. \& Wilder, F. V. The effects of vitamin C supplementation on incident and progressive knee osteoarthritis: a longitudinal study. Publ. Health Nutr. 14, 709-715 (2011)

79. Chaganti, R. K. et al. High plasma levels of vitamin C and $E$ are associated with incident radiographic knee osteoarthritis. Osteoarthritis Cartilage 22, 190-196 (2014).
80. Kraus, V. B. et al. Ascorbic acid increases the severity of spontaneous knee osteoarthritis in guinea pig model. Arthritis Rheum. 50, 1822-1831 (2004).

81. Misra, D. et al. Vitamin $\mathrm{K}$ deficiency is associated with incident knee osteoarthritis. Am. J. Med. 126 243-248 (2013).

82. Neogi, T. et al. Low vitamin $\mathrm{K}$ status is associated with osteoarthritis in the hand and knee. Arthritis Rheum. 54, 1255-1261 (2006).

83. Shea, M. K. et al. The association between vitamin K status and knee osteoarthritis features in older adults: the Health, Aging and Body Composition Study. Osteoarthritis Cartilage 23, 370-378 (2015)

84. Datta, P. et al. High-fat diet-induced acceleration of osteoarthritis is associated with a distinct and sustained plasma metabolite signature. Sci. Rep. 7 8205 (2017).

85. Mooney, R. A., Sampson, E. R., Lerea, J., Rosier, R. N. \& Zuscik, M. J. High-fat diet accelerates progression of osteoarthritis after meniscal/ligamentous injury. Arthritis Res. Ther. 13, R198 (2011).

86. Le Chatelier, E. et al. Richness of human gut microbiome correlates with metabolic markers. Nature 500, 541-546 (2013).

87. Biagi, E. et al. Through ageing, and beyond: gut microbiota and inflammatory status in seniors and centenarians. PLOS ONE 5, e10667 (2010).

88. Conlon, M. A. \& Bird, A. R. The impact of diet and lifestyle on gut microbiota and human health Nutrients 7, 17-44 (2015).

89. Brahe, L. K., Astrup, A $\delta$ Larsen, L. H. Is butyrate the link between diet, intestinal microbiota and obesityrelated metabolic diseases? Obes. Rev. 14, 950-959 (2013).

90. Russell, W. R., Hoyles, L., Flint, H. J. \& Dumas, M. E. Colonic bacterial metabolites and human health. Curr. Opin. Microbiol. 16, 246-254 (2013).

91. Boulange, C. L., Neves, A. L., Chilloux, J., Nicholson, J. K $\&$ Dumas, M. E. Impact of the gut microbiota on inflammation, obesity, and metabolic disease. Genome Med. 8, 42 (2016)

92. Huang, Z. \& Kraus, V. B. Does lipopolysaccharidemediated inflammation have a role in OA? Nat. Rev. Rheumatol. 12, 123-129 (2016).

93. Collins, K. H. et al. Relationship between inflammation, the gut microbiota, and metabolic osteoarthritis development: studies in a rat model. Osteoarthritis Cartilage 23, 1989-1998 (2015).

94. Dai, Z., Lu, N., Niu, J., Felson, D. T. \& Zhang, Y. Dietary fiber intake in relation to knee pain trajectory. Arthritis Care Res. 69, 1331-1339 (2017).

95. Dai, Z, Niu, J., Zhang, Y., Jacques, P. \& Felson, D. T. Dietary intake of fibre and risk of knee osteoarthritis in two US prospective cohorts. Ann. Rheum. Dis. 76, 1411-1419 (2017)

96. Schott, E. M. et al. Targeting the gut microbiome to treat the osteoarthritis of obesity. JCl Insight https:// doi.org/10.1172/jci.insight.95997 (2018).

97. Palmieri-Smith, R. M. et al. The role of athletic trainers in preventing and managing posttraumatic osteoarthritis in physically active populations: a consensus statement of the Athletic Trainers' Osteoarthritis Consortium. J. Athlet. Train. 52, 610-623 (2017).

98. Shaw, C. N. \& Stock, J. T. Extreme mobility in the Late Pleistocene? Comparing limb biomechanics among fossil Homo, varsity athletes and Holocene foragers. J. Hum. Evol. 64, 242-249 (2013).

99. Berger, T. D. \& Trinkaus, E. Patterns of trauma among the Neandertals. J. Archaeol. Sci. 22, 841-852 (1995)

100. Hallal, P. C. et al. Global physical activity levels: surveillance progress, pitfalls and prospects. Lancet 380, 247-257 (2012).

101. Jacka, F. N. et al. Lower levels of physical activity in childhood associated with adult depression. J. Sci. Med. Sport 14, 222-226 (2011).

102. Arsenis, N. C., You, T., Ogawa, E. F., Tinsley, G. M. \& Zuo, L. Physical activity and telomere length: impact of aging and potential mechanisms of action. Oncotarget 8, 45008-45019 (2017)

103. Weibel, E. R., Taylor, C. R. \& Hoppeler, H. The concept of symmorphosis: a testable hypothesis of structure function relationship. Proc Natl Acad Sci. USA 88 10357-10361 (1991)

104. Roos, E. M. \& Arden, N. K. Strategies for the prevention of knee osteoarthritis. Nat. Rev. Rheumatol. 12 , 92-101 (2016).

105. Slemenda, C. et al. Quadriceps weakness and osteoarthritis of the knee. Ann. Intern. Med. 127 97-104 (1997).

106. Vanwanseele, B., Eckstein, F., Knecht, H., Spaepen, A $\&$ Stussi, E. Longitudinal analysis of cartilage atrophy 
in the knees of patients with spinal cord injury. Arthritis Rheum. 48, 3377-3381 (2003).

107. Vanwanseele, B., Eckstein, F., Knecht, H., Stüssi, E. \& Spaepen, A. Knee cartilage of spinal cord-injured patients displays progressive thinning in the absence of normal joint loading and movement. Arthritis Rheum. 46, 2073-2078 (2002).

108. Urquhart, D. M. et al. What is the effect of physical activity on the knee joint? A systematic review. Med. Sci. Sports Exerc. 43, 432-442 (2011).

109. Jones, G. et al. Knee articular cartilage development in children: a longitudinal study of the effect of sex, growth, body composition, and physical activity. Pediatr. Res. 54, 230-236 (2003).

110. Racunica, T. L. et al. Effect of physical activity on articular knee joint structures in communitybased adults. Arthritis Rheum. 57, 1261-1268 (2007).

111. Leong, D. J. et al. Matrix metalloproteinase-3 in articular cartilage is upregulated by joint immobilization and suppressed by passive joint motion. Matrix Biol. 29, 420-426 (2010).
112. Nomura, M. et al. Thinning of articular cartilage after joint unloading or immobilization. An experimental investigation of the pathogenesis in mice. Osteoarthritis Cartilage 25, 727-736 (2017).

113. Paukkonen, K., Jurvelin, J. \& Helminen, H. J. Effects of immobilization on the articular cartilage in young rabbits. A quantitative light microscopic stereological study. Clin. Orthop. Relat. research, 270-280 (1986).

114. Campbell, T. M., Reilly, K., Laneuville, O., Uhthoff, H. \& Trudel, G. Bone replaces articular cartilage in the rat knee joint after prolonged immobilization. Bone 106 42-51 (2018).

115. Bricca, A., Juhl, C. B., Grodzinsky, A. J. \& Roos, E. M. Impact of a daily exercise dose on knee joint cartilage - a systematic review and meta-analysis of randomized controlled trials in healthy animals. Osteoarthritis Cartilage 25, 1223-1237 (2017)

116. Teichtahl, A. J. et al. The interaction between physical activity and amount of baseline knee cartilage. Rheumatology 55, 1277-1284 (2016)

117. Arokoski, J. P., Jurvelin, J. S., Vaatainen, U. \& Helminen, H. J. Normal and pathological adaptations of articular cartilage to joint loading. Scand. J. Med. Sci. Sports 10, 186-198 (2000).

\section{Acknowledgements}

The work of the authors is financially supported by grants from the French Society of Rheumatology, Fondation Arthritis (ROAD network) (to F.B.), the Hintze Family Charitable Foundation, the American School of Prehistoric Research (Harvard University) (to D.E.L.) and a grant from the US National Institutes of Health (AR47785 to D.T.F.).

\section{Author contributions}

All authors researched data for the article, wrote the article, made substantial contribution to discussions of the content and reviewed and/or edited the manuscript before submission.

Competing interests

The authors declare no competing interests.

Publisher's note

Springer Nature remains neutral with regard to jurisdictional claims in published maps and institutional affiliations. 